\title{
Erysipelas in Tunisian Patients: Epidemiological, Clinical Features and Risk Factors in Internal Medicine
}

\author{
Kechaou*, Ben Haj Yahya M, Cherif E, Boukhris I and Ben Hassine L \\ Department B of Internal Medicine, Hospital Charles Nicolle, Faculty of Medicine, University of Tunis El Manar, Tunisia.
}

Received: May 30, 2018; Published: June 11, 2018

*Corresponding author: Kechaou Ines, Department B of Internal Medicine, Charles Nicolle Hospital, Boulevard 9 April 1938. 1080. Tunis, Tunisia

\begin{abstract}
Background: The purpose of our work was to study the epidemiological, clinical features and risk factors of erysipelas in Internal Medicine.

Methods: Retrospective study including 86 patients with erysipelas collected at our department of internal medicine between 2005 and 2016.

Results: There were 44 men and 42 women. The average age of our patients was 57.77. Erysipelas mainly affected the lower limbs in $95.3 \%$ of cases. Fever was present in $47.67 \%$ of cases. General underlying conditions were dominated by diabetes (55.8\%), obesity (37.2\%) and overweight (31.4\%). Loco-regional favoring factors were essentially represented by lymphedema (18.6\%), venous insufficiency (12.8\%) and arteritis of the lower limbs (12.8\%). A local site of entry was identified in $83.7 \%$ of the cases. The average number of total favorable factors was 3.12. Laboratory findings revealed leukocytosis in $66.3 \%$ of cases and CRP elevation in $77.6 \%$ of the cases. Bacteriological investigations identified gram negative bacilli in 3 patients: on blood cultures (Klebsiella Pneumoniae) and samples from needle aspirate (Enterobacter Cloacae and Serratia Marcescens). Erysipelas was recurrent in 45.34\% of cases. Risk factors for recurrence were mycosis and lymphedema.
\end{abstract}

Conclusion: Prevention of erysipelas is essentially based on the treatment of local and general factors. Clinicians must be aware to the possibility of other causative microorganisms like GNB in front of erysipelas.

Keywords: Erysipelas; Risk Factor; Microbiology; Gram Negative Bacilli; Recurrence

\section{Introduction}

Erysipelas is known to be an acute non-necrotizing bacterial dermohypodermitis mainly due to group A beta hemolytic streptococci. In Internal Medicine, the main difficulty is to identify patients at risk of getting worse because many of our patients are immunocompromised. Another challenge in our practice is to identify the causative organisms in case of non-response to classic treatment. The purpose of our work was to study the epidemiological, clinical and risk factors of erysipelas.

\section{Patients}

We conduct a retrospective study including 86 patients with erysipelas collected at Tunisian department of internal medicine between 2005 and 2016. We included in our study the files of hospitalized patients for erysipelas admitted by the emergencies or the external consultation. The diagnosis of erysipelas was made in the presence of an erysipelatoid edematous placard, well limited, surrounded or not by a peripheral bead associated or not with general signs. We excluded from our study all erythematous rashes that did not relate to erysipelas and complications such as cellulitis and necrotizing fasciitis.

\section{Methods}

Epidemiological and clinical parameters collected included age, sex, hospital stay, relapses, cases with outpatient and inpatient treatment respectively, localization of erysipelas, antibiotic treatment and duration, the criteria of sepsis, $\mathrm{C}$ - reactive protein (CRP), white blood count (WBC), creatinine, and platelet levels on admission [1]. Underlying disease were collected records. Contributing factors were classified in general and loco regional predisposing factors. The sites of entry have been specified. Microbiological results from blood cultures and local samples were collected from our Laboratory for Medical Microbiology in our hospital. We have studied the risk factors for recurrence among our patients based on socio-demographic, general, locoregional factors and the sites of entry. For that we compared those with recurrent erysipelas to those without recurrence. 


\section{Statistical Analysis}

The data were analyzed using SPSS version 19 software. For the descriptive study, simple frequencies were calculated for the qualitative variables. Averages, medians and standard deviations were calculated for the quantitative variables. The comparison of 2 averages was done using Student's t-test. The comparison of the percentages on independent series was carried out by the Pearson Chi2 test and in case of non-validity by the exact test of Fischer. The threshold of significance was set at 0.05 .

\section{Results}

\section{Epidemiological characteristics}

There were 44 men and 42 women (sex ratio: 1.04). The average age of our patients was $57.77 \pm 15.65$ years ( 23 - 87 years). Comorbidities in our patients were dominated by diabetes (55.8\%), hypertension (52.3\%) and chronic renal failure with renal clearance of creatinine less than $60 \mathrm{ml} / \mathrm{mn}$ (44.2\%). Hypersensitivity to penicillin and autoimmune disease was observed in respectively 8.1\%. Erysipelas occurred during the autumn-summer period in $63.9 \%$ of cases.

\section{Clinical Study}

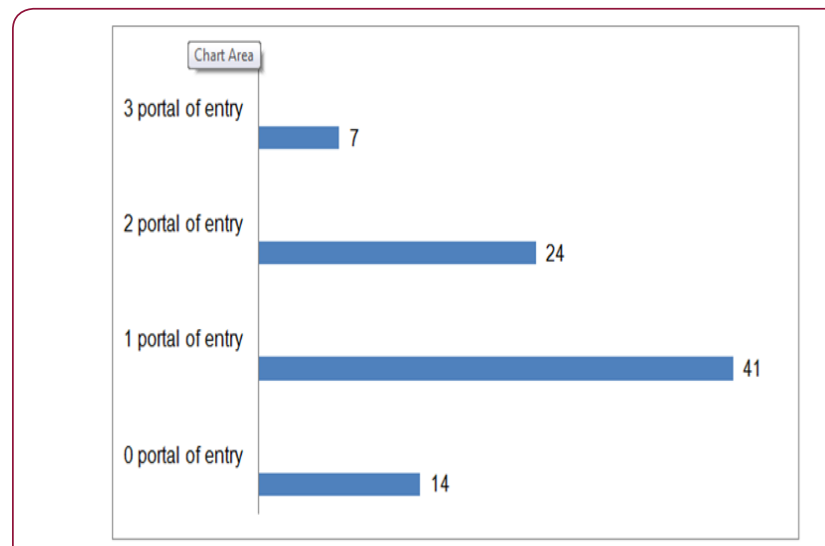

Figure 1: distribution of patients according to portal of entry.

The average of diagnostic delay was 9.94 days. Erysipelas affected the lower limbs in 82 cases (95.3\%) and was bilateral in 9.3\%. Upper limbs were involved in the other 4 cases. At the time of admission, fever was present in 41 patients (47.67\%). Loco regional manifestations observed in our study were: inguinal lymphadenopathy $(24.4 \%)$, phlyctena $(15.1 \%)$, bubbles $(8.1 \%)$, peripheral bead $(3.5 \%)$, vesicles $(3.5 \%)$, purpura $(2.3 \%)$ and pustules (1.2\%). A history of erysipelas was found in $43 \%$ of cases with an average number of episodes of $0.97 \pm 1.6(0-10)$. General underlying conditions were diabetes $(55.8 \%)$, obesity $(37.2 \%)$, overweight (31.4\%), ethylism (16.3\%), not decompensated heart failure $(9.3 \%)$, long-term oral corticosteroids $(4.7 \%)$, immunosuppressive therapy with azathioprine $(1.2 \%)$ and postalcoholic cirrhosis (1.2\%). Loco-regional favoring factors were: lymphedema (18.6\%), venous insufficiency (12.8\%), arteritis of the lower limbs (12.8\%) and erysipelas on irradiated area (1.2\%). A local site of entry was identified in $83.7 \%$ of the cases. It was essentially represented by mycosis (74.4\%) and toe web intertrigo
(72.1\%). The others were: onychomycosis (18.6\%), traumatic skin wound (10.4\%), plantar hyperkeratosis (10.4\%), diabetic foot ulcer (7\%), leg ulcer (4.7\%), eczema (4.7\%), wet gangrene $(2.3 \%)$ and sting by foreign body (1.2\%). At least one site of entry was found in 72 patients $(83.7 \%)$ (Figure 1). The average number of total favorable factors was $3.12 \pm 1.5(0-8)$ (Table 1$)$.

Table 1: Distribution of patients according to the number of contributing factors to erysipelas.

\begin{tabular}{|c|c|c|}
\hline $\begin{array}{c}\text { Number of Favorable } \\
\text { Factors }\end{array}$ & $\begin{array}{c}\text { Number of } \\
\text { Patients } / 86\end{array}$ & Percentage (\%) \\
\hline 0 & 4 & $4.7 \%$ \\
\hline 1 & 7 & $8.1 \%$ \\
\hline 2 & 19 & $22.1 \%$ \\
\hline 3 & 25 & $29.1 \%$ \\
\hline 4 & 16 & $18.6 \%$ \\
\hline 5 & 8 & $9.3 \%$ \\
\hline 6 & 6 & $7 \%$ \\
\hline 8 & 1 & $1,2 \%$ \\
\hline
\end{tabular}

\section{Laboratory Data}

Laboratory findings revealed leukocytosis in 66.3\% (Figure 2) and CRP elevation in $77.6 \%$ (Figure 3 ).

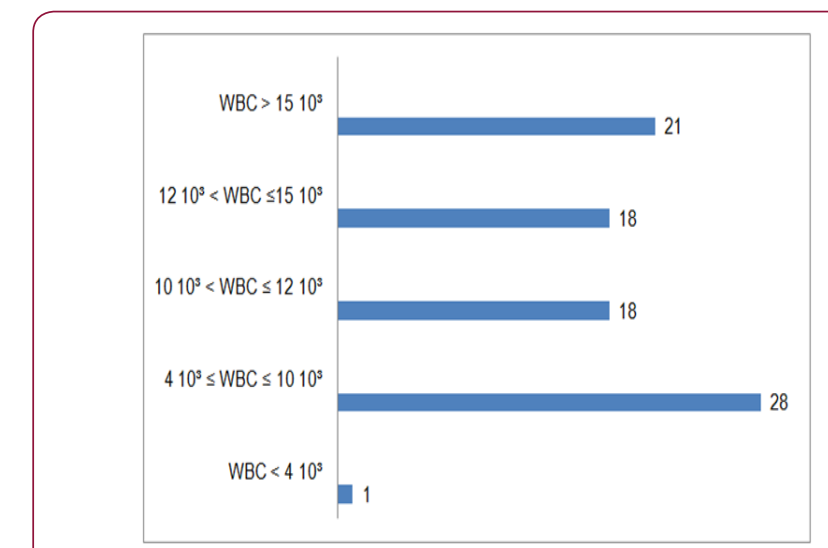

Figure 2: Distribution of patients according to the value of white blood count (WBC).

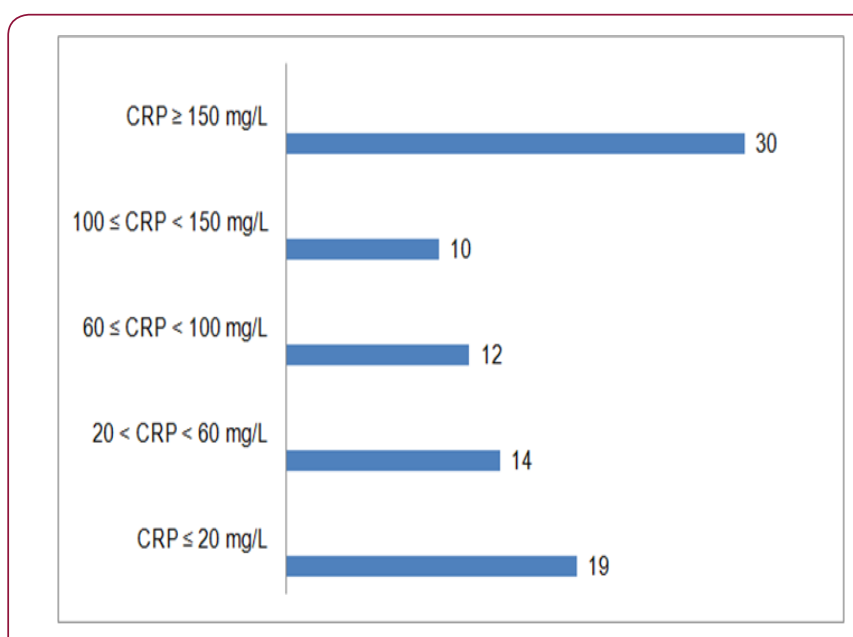

Figure 3: Distribution of patients according to the value of CRP. 


\section{Microbiology}

Blood cultures were made in patients with fever. Samples from needles aspirate was made in cases who didn't respond to empiric antibiotic. Bacteriological investigations identified gram negative bacilli (GNB) in 3 diabetic patients: on blood cultures (Klebsiella Pneumoniae) and samples from needle aspirate (Enterobacter Cloacae and Serratia Marcescens). Staphylococcus was not isolated in our study. But, it was suspected in patients with cutaneous effraction, pustules or abscess.

\section{Treatment}

In 17 cases $(19.7 \%)$, empirical treatment was initiated with pristinamycin (10.5\%), benzyl penicillin (6.9\%) and amoxicillin with clavulanic acid (2.3\%). In the others 69 patients who received antibiotherapy prior to admission, 43 received amoxicillin and clavulanic acid, 17 benzyl penicillin and 9 pristinamycin. The treatment was readjusted according to antibiogram in the GNB infections. The median duration of antibiotherapy was 15 days.

\section{Evolution and Complications}

The delay in obtaining apyrexia was 1.55 days \pm 2.1 ( 0 to 9 days). Local improvement of erythema was obtained after an average delay of $4.9 \pm 2.6$ days ( $1-12$ days). Erysipelas was complicated by deep vein thrombosis $(n=8)$, diabetic ketosis $(n=5)$, abscess $(n=2)$ and severe sepsis $(n=1)$. Erysipelas was recurrent in $45.34 \%$ of cases. The average number of recurrence was $1.98 \pm 1.58$ (1 - 11).

\section{Risk Factors for Recurrence}

Risk factors for recurrence were mycosis and lymphedema ( $p<0.05$ ). The mean number of total contributing factors was significantly more common in recurrent erysipelas compared to those without recurrence $(3.67 / 2.66)(\mathrm{p}<0.05)$.

\section{Discussion}

This is a monocentric Tunisian retrospective study that has investigated epidemiological, clinical and etiological features of erysipelas as well as the peculiarities of therapeutic management. This study allowed a retrospective evaluation of our practices in case of erysipelas in internal medicine. It showed a great variability in predisposing factors, sites of entry and complications of erysipelas. In our study as in the literature, the general contributing factors were dominated by diabetes, obesity and alcoholism and the main local predisposing factors were lymphedema and venous insufficiency [2-6]. In front of erysipelas, looking for sites of entry must be systematic. In our study, the most common site of entry was mycosis. As reported by Mokni et al, any disruption of cutaneous barrier was the most significant risk factor followed by leg edema [7]. Acting on contributing factors may be an interesting alternative to avoid recurrences.

Although erysipelas is a common pathology, identifying a causative agent is rare. Despite beta-hemolytic streptococci is the most common causative agent, it was isolated rarely $[8,9]$. In addition to streptococcus, the second causative agent responsible of erysipelas was staphylococcus aureus [9]. In our study, it was not identified directly but it was suspected when there was a cutaneous abrasion or abscess. In our patients, blood cultures and samples from needle aspirate identified 3 GNB. As reported by several authors, cultures from needle aspirates or punch biopsies of the inflamed skin identify pathogenic bacteria in a minority of cases $[8,9]$. GNB have been reported rarely in erysipelas in comparison with complications due to the whole soft tissue infections [9-15]. It is therefore important to multiply the bacteriological samples to find the causal agent especially when we expect no improvement under classic treatment. In our study, benzyl penicillin was not the most used treatment because of the risk of heart failure in hypertensive patients and in those with chronic renal failure and the risk of cardiac decompensation in patients with a history of heart failure.

We have studied the risk factors for recurrence among our patients based on socio-demographic, general, locoregional factors and the sites of entry. We found, that the presence lymphedema and mycosis were risk factors of recurrence in our patients. We also found that the mean number of total contributing factors was significantly more common in recurrent erysipelas compared to those without recurrence $(3.67 / 2.66)$. Our study joins data from the literature concerning lymphoedema and mycoses. While for other risk factors advanced in the literature as the antecedent of erysipelas, ethylism, obesity, overweight, heart failure, venous insufficiency, arteritis of the lower limbs, post-traumatic wounds, they were not significant in our study probably because of the small size in our study [16-18]. Finally, the retrospective nature of our study remains the main limiting factor.

\section{Conclusion}

Erysipelas is a common pathology. Prevention is essentially based on the treatment of local and general factors. Other causative agents may be suspected in cases with systemic or local complications.

\section{References}

1. Edward A (2016) New Definitions for Sepsis and Septic Shock. J Am Med Assoc 315(8): 757-759.

2. Zaraa I, Zeglaoui F, Zouari B, Ezzine N, Fazaa B, et al. (2004) Erysipelas: retrospective study of 647 patients. Tunis Med 82(11): 990-995.

3. Ben Fredj I, Sehli J, Essid A, Hadj Khelifa S, Mrad B, et al. (2008) Dermohypodermites aiguës infectieuses non nécrosantes des membres: à propos de 196 cas. Rev Med Int 29(1): S94.

4. Amal S, Houass S, Laissaoui K, Moufid K, Trabelsi M (2004) Érysipèle. Profil épidémiologique, clinique et évolutif dans la région de Marrakech (100 observations). Med Mal Infect 34(4): 171-176.

5. Lanoux P, Penalba Ch, Legin C, Reveil JCl (1992) L'érysipèle: à propos de cent-dix-huit observations. Rev Med Int 13(6): S232.

6. Mrouki M, Souissi A, Derbel F, Karray M, Jellouli A, et al. (2016) Caractéristiques épidémiologiques et cliniques de l'érysipèle dans la population des agents des forces de l'intérieur. Ann Dermatol Venerol 143(4S1): S39.

7. Mokni M, Dupuy A, Denguezli M, Dhaoui R, Bouassida S, et al. (2006) Risk factors for erysipelas of the leg in Tunisia: a multicenter casecontrol study. Dermatology 212(2): 108-112.

8. Eriksson B, Jorup-Rönström C, Karkkonen K, Sjöblom AC, Holm SE (1996) Erysipelas: clinical and bacteriologic spectrum and serological aspects. Clin Infect Dis 23(5): 1091-1098. 
9. Bläckberg A, Trell K, Rasmussen M (2015) Erysipelas, a large retrospective study of aetiology and clinical presentation. BMC Infectious Diseases 15: 402.

10. Caetano M, Amorim I (2005) Erisipela. Acta Med Port 18: 385-394.

11. Ruef C (2008) Complicated skin and soft-tissue infection-consider Gram-negative pathogens. Infection 36(4): 295.

12. Lazzarini L, Conti E, Tositti G, De Lalla F (2005) Erysipelas and cellulitis: clinical and microbiological spectrum in an Italian tertiary care hospital. J Infect 51(5): 383-389.

13. Chang CM, Lee HC, Lee NY, Lee IW, Wu CJ, et al. (2008) Communityacquired Klebsiella pneumonia complicated skin and soft-tissue infections of extremities: Emphasis on cirrhotic patients and gas formation. Infection 36(4): 328-334.

14. Moet GJ, Jones RN, Biedenbach DJ, Stilwell MG, Fritsche TR (2007) Contemporary causes of skin and soft tissue infections in North America,
Latin America, and Europe: Report from the SENTRY antimicrobial surveillance program (1998-2004). Diagn Microbiol Infect Dis 57(1): 7-13.

15. Dalal A, Eskin Schwartz M, Mimouni D, Ray S, Days W, et al. (2017) Interventions for the prevention of recurrent erysipelas and cellulitis (Review). Cochrane Database of Systematic Reviews 6: CD009758.

16. Inghammar M, Rasmussen M, Linder A (2014) Recurrent erysipelas-risk factors and clinical presentation. BMC Infect Dis 14: 270.

17.S Leclerc A, Teixeira E, Mahé V, Descamp B, Crickx 0, et al. (2007) Recurrent erysipelas: 47 cases. Dermatology 214(1): 52-57.

18. Pavlosky F, Amrani S, Trau H (2004) Reccurent erysipelas: risk factors. JDDG 2(2): 89-95. (c) (1) This work is licensed under Creative

Submission Link: https://biomedres.us/submit-manuscript.php

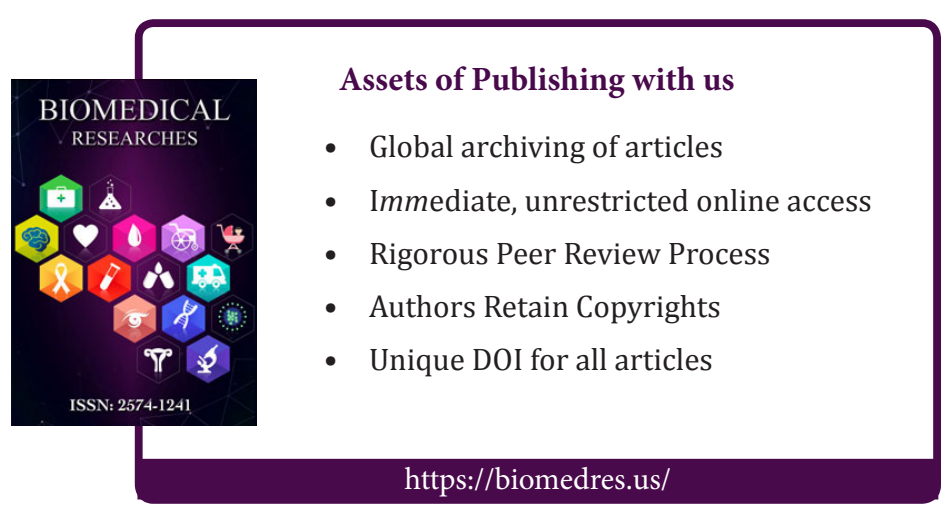

